\title{
Análise da transição entre dias secos e chuvosos por meio da cadeia de Markov de terceira ordem
}

\author{
Thadeu Keller Filho(1), Jurandir Zullo Junior ${ }^{(2)}$ e Paulo Roberto Schubnell de Rezende Lima ${ }^{(3)}$
}

\begin{abstract}
(1)Pontifícia Universidade Católica do Rio de Janeiro, Rua Marquês de São Vicente, no 225, Sala 210 F, CEP 22453-900 Rio de Janeiro, RJ. E-mail: thadeu@econ.puc-rio.br (2)Universidade Estadual de Campinas, Centro de Pesquisas Meteorológicas e Climáticas Aplicadas à Agricultura, Cidade Universitária Zeferino Vaz, CEP 13083-970 Campinas, SP. E-mail: jurandir@cpa.unicamp.br (3)Estocástica Consultoria e Serviços Técnicos em Estatística Ltda., SQS 314, Bloco D, apto. 502, CEP 70383-040 Brasília, DF. E-mail: paulo.schubnell@yahoo.com.br
\end{abstract}

Resumo - O objetivo deste trabalho foi verificar se as ocorrências de dias secos e chuvosos são condicionalmente dependentes da seqüência dos três dias secos e chuvosos anteriores, numa zona pluviometricamente homogênea, por meio da cadeia não-homogênea de Markov de terceira ordem. Os resultados mostraram que as probabilidades diárias de transição podem ser adequadamente estimadas, com base em dados agregados bimestralmente, seguidas de interpolação por meio de funções sinusoidais. Além disso, evidenciou-se que, naquela zona, as ocorrências diárias de chuva são condicionalmente dependentes da seqüência de dias secos e chuvosos nos três dias anteriores. A cadeia não-homogênea de Markov de terceira ordem é um importante instrumento para a análise da dependência entre as seqüências de dias secos e chuvosos em determinadas regiões.

Termos para indexação: zonas pluviometricamente homogêneas, ocorrência de chuva, probabilidade de transição.

\section{Analysis of the transition between dry and wet days through third-order Markov chains}

\begin{abstract}
The aim of this work was to verify if the occurrence of dry and wet days are conditionally dependent on the sequences of the dry and wet three preceding days, in a rainfall homogeneous area, using the nonhomogeneous third-order Markov chains. The results showed that daily transition probabilities can be properly estimated from two-month aggregate data, and then adjusted by means of sinusoidal functions. Besides, it was evidenced that everyday rain events in that area are conditionally dependent on the sequences of the dry and wet three days previous to occurrences. The third-order nonhomogeneous Markov chains are an important instrument for the analysis of the dependence between sequences of dry and wet days in certain areas.
\end{abstract}

Index terms: rainfall homogeneous areas, rainfall occurrence, transition probabilities.

\section{Introdução}

A distribuição e a irregularidade da chuva, no tempo e no espaço, são as principais características climáticas de uma região. A precipitação pluvial, assim como outros fatores climáticos, é sujeita a grandes variações, que afetam de modo significativo a produção agrícola.

A produtividade agrícola é fortemente influenciada pela oferta pluviométrica e pela freqüência e duração dos períodos secos. Na maior parte das regiões tropicais, a alternância entre períodos chuvosos e secos apresenta um notável comportamento sazonal. A agricultura, conforme Ayode (1983), é praticada com maior êxito durante a estação chuvosa ou com o uso da irrigação durante a estação seca.
Em algumas regiões, principalmente no Cerrado, a precipitação total do período chuvoso é suficiente para o desenvolvimento da agricultura, porém, é comum a ocorrência de seqüências de dias secos durante a estação chuvosa; são breves períodos conhecidos como veranicos (Souza \& Peres, 1998). O estudo da ocorrência de veranicos é de grande importância para a agricultura pois, geralmente, esses breves períodos de estiagem atingem a cultura em sua fase reprodutiva, o que causa redução em sua produtividade (Assad et al., 1993).

A determinação da distribuição de probabilidades da precipitação pluvial e a estimação das probabilidades da transição entre dias secos e chuvosos são de grande importância para a otimização do planejamento das atividades agrícolas, pois contribuem de forma decisiva para 
a tomada de decisão quanto às épocas de plantio, para a avaliação do risco de deficiência hídrica e para o dimensionamento e manejo dos sistemas irrigados.

O principal instrumento na análise probabilística das transições entre dias secos e chuvosos é a cadeia de Markov (Gordon, 1965), amplamente utilizada no estudo da ocorrência da precipitação pluvial diária, tanto no exterior quanto no Brasil.

Em um trabalho pioneiro, Gabriel \& Neumann (1962) utilizaram cadeias homogêneas de Markov para descrever a alta persistência de dias secos consecutivos em Tel Aviv, Israel. Outro trabalho pioneiro foi o de Stern \& Coe (1982), que definiram um modelo de simulação para a precipitação pluvial diária, no qual a ocorrência de dias chuvosos é gerada por uma cadeia de Markov, e a quantidade de chuva é gerada por uma distribuição gama. Esse modelo foi aperfeiçoado, utilizando-se séries de Fourier para representar as probabilidades de transição (Stern \& Coe, 1984).

A partir desses trabalhos, multiplicaram-se as aplicações de cadeias de Markov na análise estatística da ocorrência de dias secos e úmidos. Entre as inúmeras pesquisas recentes, pode-se citar: Muhammad \& Nabi (1991), que analisaram a ocorrência de chuva em quatro localidades no Paquistão; Assis (1991), que modelou a ocorrência de dias secos e úmidos em Piracicaba, SP, e Pelotas, RS; Back (1997), que se baseou nos modelos de Stern \& Coe $(1982,1984)$, para ajustar a precipitação pluvial no posto de Urussanga, SC; Punyawardena \& Kulasiri (1997), que compararam modelos de cadeias de Markov de primeira e de segunda ordem para a descrição da ocorrência semanal de chuva, na zona seca de Sri Lanka; De Groen \& Savenije (2000), que utilizaram cadeias de Markov para simular a ocorrência de chuva, em um modelo de transpiração mensal, com dados de três localidades no Zimbábue; Andrade Júnior et al. (2001), que simularam a precipitação pluvial diária para Parnaíba e Teresina, PI, com cadeias de Markov para gerar seqüências de dias secos e úmidos.

Na maioria dos estudos se utiliza a cadeia de Markov de primeira ou de segunda ordem. Todavia, o uso de cadeias de Markov de ordem superior a dois é de grande importância, pois, em certas regiões, a ocorrência de chuva em um dia pode ser condicionalmente dependente da seqüência de dias secos e chuvosos em vários dias anteriores. Isto pode acontecer, por exemplo, em regiões onde, em certas épocas, é freqüente a ocorrência de vários dias consecutivos de chuva ou longas séries de dias secos.
O objetivo deste trabalho foi verificar se as ocorrências de dias secos e chuvosos são condicionalmente dependentes da seqüência de dias secos e chuvosos nos três dias anteriores, em uma zona pluviometricamente homogênea, por meio de cadeias não-homogêneas de Markov de terceira ordem.

\section{Material e Métodos}

O processo gerador de uma seqüência aleatória de dias secos e chuvosos pode ser descrito por uma cadeia de Markov, com as seguintes características:

1) estados da cadeia: $S$ = dia seco; $C$ = dia chuvoso;

2) indicador do estado no dia t:

$X_{t}=0$ se ocorre $S$ no dia $t$; $X_{t}=1$ se ocorre $C$ no dia $t$

3) probabilidades de transição de estado:

$\mathrm{P}\left(\mathrm{J}_{\mathrm{t}} / \mathrm{J}_{\mathrm{t}-\mathrm{r}}, \mathrm{J}_{\mathrm{t}-\mathrm{r}+1}, \ldots, \mathrm{J}_{\mathrm{t}-1}\right)=\mathrm{P}\left(\mathrm{X}_{\mathrm{t}}=\mathrm{J}_{\mathrm{t}} / \mathrm{X}_{\mathrm{t}-\mathrm{r}}=\mathrm{J}_{\mathrm{t}-\mathrm{r}}, \mathrm{X}_{\mathrm{t}-\mathrm{r}+1}\right.$

$\left.=\mathrm{J}_{\mathrm{t}-\mathrm{r}+1}, \ldots, \mathrm{X}_{\mathrm{t}-1}=\mathrm{J}_{\mathrm{t}-1}\right)$

em que $\mathrm{r}(\mathrm{r}=1,2,3, \ldots)$ é a ordem da cadeia, e $\mathrm{J}_{\mathrm{t}}$ é o valor do indicador do estado no dia t.

Assim, $\mathrm{P}(0 / 001)$ representa a probabilidade de que um dia seja seco, na hipótese de que os três dias anteriores tenham sido, seqüencialmente, seco, seco e chuvoso.

Como, nesse modelo, as probabilidades de transição não são constantes, pois variam com o dia t, a cadeia de Markov é não-homogênea.

Uma cadeia de Markov é de ordem $r(r=1,2,3, \ldots)$, se for:

$\mathrm{P}\left(\mathrm{J}_{\mathrm{t}} / \mathrm{J}_{\mathrm{t}-\mathrm{r}}, \mathrm{J}_{\mathrm{t}-\mathrm{r}+1}, \ldots, \mathrm{J}_{\mathrm{t}-1}\right)=\mathrm{P}\left(\mathrm{J}_{\mathrm{t}} / \mathrm{J}_{\mathrm{t}-\mathrm{r}-1}, \mathrm{~J}_{\mathrm{t}-\mathrm{r}}, \mathrm{J}_{\mathrm{t}-\mathrm{r}+1}, \ldots, \mathrm{J}_{\mathrm{t}-1}\right)$ quaisquer que sejam $\mathrm{J}_{\mathrm{t}-\mathrm{r}-1}, \mathrm{~J}_{\mathrm{t}-\mathrm{r}}, \mathrm{J}_{\mathrm{t}-\mathrm{r}+1}, \ldots, \mathrm{J}_{\mathrm{t}-1}, \mathrm{~J}_{\mathrm{t}}(\mathrm{r}=1,2,3, \ldots)$.

Como os indicadores $\mathrm{J}_{\mathrm{t}}$ assumem apenas os valores 0 e 1 , a igualdade (3) implica:

$\mathrm{P}\left(1-\mathrm{J}_{\mathrm{t}} / \mathrm{J}_{\mathrm{t}-\mathrm{r}}, \mathrm{J}_{\mathrm{t}-\mathrm{r}+1}, \ldots, \mathrm{J}_{\mathrm{t}-1}\right)=\mathrm{P}\left(1-\mathrm{J}_{\mathrm{t}} / \mathrm{J}_{\mathrm{t}-\mathrm{r}-1}, \mathrm{~J}_{\mathrm{t}-\mathrm{r}}, \mathrm{J}_{\mathrm{t}-\mathrm{r}+1}, \ldots, \mathrm{J}_{\mathrm{t}-1}\right)$.

Confrontando-se (3) e (4), podemos concluir que uma cadeia de Markov é de ordem $\mathrm{r}(\mathrm{r}=1,2,3, \ldots)$ se:

$\mathrm{P}\left(0 / \mathrm{J}_{\mathrm{t}-\mathrm{r}}, \mathrm{J}_{\mathrm{t}-\mathrm{r}+1}, \ldots, \mathrm{J}_{\mathrm{t}-1}\right)=\mathrm{P}\left(0 / \mathrm{J}_{\mathrm{t}-\mathrm{r}-1}, \mathrm{~J}_{\mathrm{t}-\mathrm{r}}, \mathrm{J}_{\mathrm{t}-\mathrm{r}+1}, \ldots, \mathrm{J}_{\mathrm{t}-\mathrm{1}}\right)$, quaisquer que sejam $\mathrm{J}_{\mathrm{t}-\mathrm{r}-1}, \mathrm{~J}_{\mathrm{t}-\mathrm{r}}, \mathrm{J}_{\mathrm{t}-\mathrm{r}+1}, \ldots, \mathrm{J}_{\mathrm{t}-1}(\mathrm{r}=1,2,3, \ldots)$.

Convém definir, ainda, cadeias de Markov de ordem zero, correspondentes a seqüências não-correlacionadas de dias secos e úmidos. Uma cadeia de Markov é de ordem zero se:

$\mathrm{P}\left(\mathrm{X}_{\mathrm{t}}=\mathrm{J}_{\mathrm{t}}\right)=\mathrm{P}\left(\mathrm{X}_{\mathrm{t}-1}=\mathrm{J}_{\mathrm{t}}\right)$, qualquer que seja $\mathrm{t}$.

Para se estimarem as probabilidades de transição, em uma cadeia de Markov de ordem r, são necessários dados referentes ao número de ocorrências de todos os $2^{\mathrm{r}+1}$ estados possíveis, em $\mathrm{r}+1$ dias consecutivos. Assim, representando-se por $\mathrm{N}_{\mathrm{n}}\left(\mathrm{J}_{\mathrm{t}-\mathrm{r}}, \mathrm{J}_{\mathrm{t}-\mathrm{r}+1}, \ldots, \mathrm{J}_{\mathrm{t}}\right)$ o número de ocorrência da seqüência de estados $\left(\mathrm{J}_{\mathrm{t}-\mathrm{r}}, \mathrm{J}_{\mathrm{t}-\mathrm{r}+1}, \ldots, \mathrm{J}_{\mathrm{t}}\right)$, em 
n dias consecutivos, as estimativas das probabilidades associadas à cadeia de Markov são dadas por:

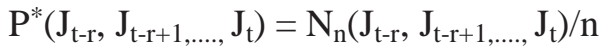

$\mathrm{P}^{*}\left(\mathrm{~J}_{\mathrm{t}} / \mathrm{J}_{\mathrm{t}-\mathrm{r}}, \mathrm{J}_{\mathrm{t}-\mathrm{r}+1}, \ldots ., \mathrm{J}_{\mathrm{t}-1}\right)=\mathrm{P}^{*}\left(\mathrm{~J}_{\mathrm{t}-\mathrm{r}}, \mathrm{J}_{\mathrm{t}-\mathrm{r}+1}, \ldots . ., \mathrm{J}_{\mathrm{t}-2}, \mathrm{~J}_{\mathrm{t}-1}, \mathrm{~J}_{\mathrm{t}}\right) / \mathrm{P}^{*}\left(\mathrm{~J}_{\mathrm{t}-\mathrm{r}}\right.$, $\left.\mathrm{J}_{\mathrm{t}-\mathrm{r}+1}, \ldots, \mathrm{J}_{\mathrm{t}-2}, \mathrm{~J}_{\mathrm{t}-1}\right)$.

Uma dificuldade para a estimação dessas probabilidades é a insuficiência de dados. De fato, o número de observações disponíveis da precipitação pluvial, em determinado dia, coincide com o número de anos abrangidos pela série histórica correspondente. Como o número de estados possíveis das seqüências $\left(\mathrm{J}_{\mathrm{t}-\mathrm{r}}, \mathrm{J}_{\mathrm{t}-\mathrm{r}+1}, \ldots, \mathrm{J}_{\mathrm{t}}\right)$ aumenta exponencialmente com a ordem da cadeia de Markov, os dados disponíveis nem sempre são suficientes, para que as estimativas (7) e (8) sejam efetuadas com precisão, se a ordem da cadeia de Markov é maior que três. Por essa razão, as análises deste trabalho limitam-se a cadeias de Markov definidas até a terceira ordem.

A escassez de dados pode ser contornada mediante a agregação espacial dos dados, agrupados em regiões onde o regime pluviométrico possui comportamento assemelhado. A regionalização explora ao máximo as informações existentes, o que permite melhorar as estimativas baseadas em dados insuficientes (Tucci, 2004).

Neste trabalho, a agregação espacial dos dados pluviométricos teve por base os estudos realizados por Keller Filho (1998) e Lima (1998) e os resultados obtidos por Keller Filho et al. (2005), que identificaram 25 zonas pluviometricamente homogêneas, em todo o território brasileiro. Foi escolhida uma dessas zonas homogêneas, que Keller Filho et al. (2005) denominaram Zona A, que abrange o norte do Tocantins, parte do sul de Goiás e grande parte de Mato Grosso. Essa zona, inserida no bioma Cerrado, apresenta uma precipitação pluvial anual relativamente elevada, com chuvas no verão e estiagens prolongadas no inverno.

Foram utilizados dados da precipitação pluvial diária, observada em cada um dos 82 postos pluviométricos da Zona A, cuja distribuição espacial é mostrada na Figura 1. Os dados abrangem um período de 15 a 30 anos e foram obtidos no banco de dados do Sistema de Monitoramento Agroclimatológico (Agritempo), desenvolvido pela Embrapa Informática Agropecuária e pela Unicamp. No Sistema Agritempo, esses dados foram validados e tiveram sua qualidade e consistência analisadas, segundo metodologia descrita por Lima (2003). A definição de dia seco corresponde àquele em que a precipitação pluvial foi inferior a $0,1 \mathrm{~mm}$.
Analisando-se as proporções observadas de dias secos na Zona A, em cada um dos 365 dias de um ano, verificase que elas evoluem de forma bastante regular e apresentam um único pico no intervalo anual, sem oscilações secundárias (Figura 2). Isto sugere que as estimativas das probabilidades de transição entre dias secos e chuvosos, ao longo de um ano, possam ser ajustadas por uma função periódica do tipo sinusoidal (Bloomfield, 1976), de periodicidade igual a 365 dias:

$\mathrm{P}_{\mathrm{t}, \mathrm{r}}=\alpha+\beta \cos (2 \pi \mathrm{t} / 365)+\gamma \operatorname{sen}(2 \pi \mathrm{t} / 365)$,

em que $\alpha, \beta$ e $\gamma$ são constantes; $t=$ dia do ano $(t=1,2,3$, 365) e

$P_{t, r}= \begin{cases}P\left(J_{t}\right) & r=0 \\ P\left(J_{t} / J_{t-r}, J_{t-r}+1, \ldots, J_{t-1}\right) & r \geq 1\end{cases}$

A regularidade da evolução das proporções de dias secos e chuvosos permite aumentar a precisão das estimativas das probabilidades de transição, mediante agregação temporal dos dados (Kendall, 1976). Verificouse que diversas sequiências possíveis dos estados de chuva e seca, em dois ou três dias consecutivos, são relativamente raras em períodos inferiores a dois meses. Por isso, serão analisadas apenas agregações em períodos não inferiores a um bimestre.

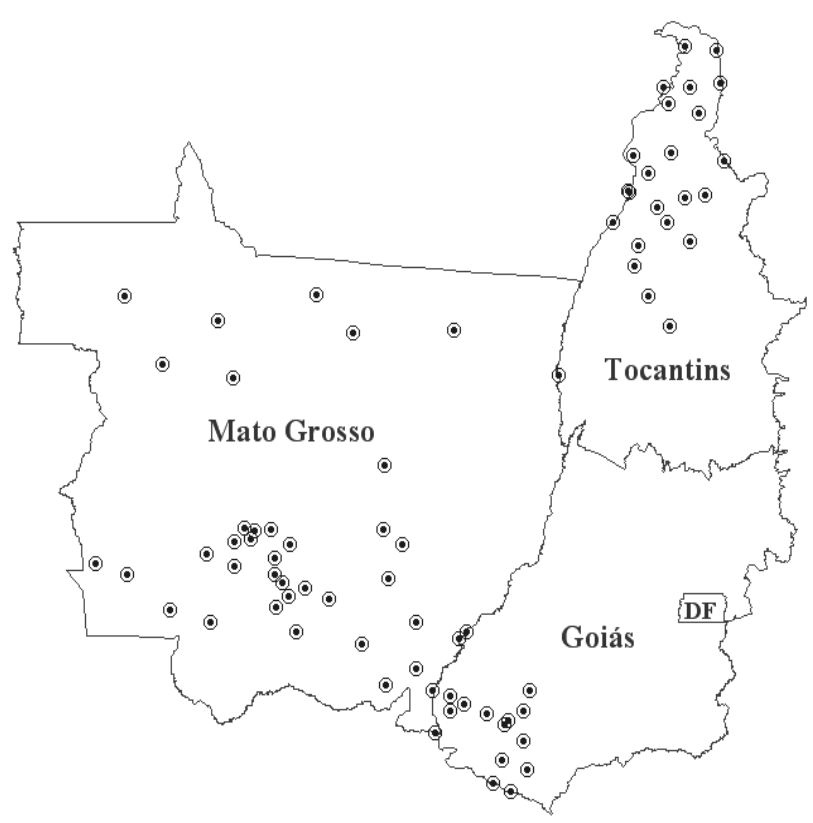

Figura 1. Distribuição espacial dos postos pluviométricos na Zona Homogênea A. 
O critério de escolha foi o de encontrar um período tão longo quanto possível, dentro do qual as probabilidades de transição não apresentem grandes discrepâncias, a fim de se obter adequado grau de precisão nas estimativas dessas probabilidades. Para esse propósito, todas as probabilidades de transição referentes a cadeias de Markov até a terceira ordem foram estimadas com base em agregações sucessivas em períodos bimestrais, trimestrais, quadrimestrais, semestrais e anuais. Utilizouse, a seguir, a análise de variância para testar a igualdade das probabilidades de transição, dentro de cada período de agregação. Para identificar, aproximadamente, as variações das probabilidades de transição, dentro dos períodos, cada um deles foi subdividido em vários grupos de meses, formados por aqueles que ocupam a mesma ordem dentro de cada período de agregação (Tabela 1). A análise de variância também permitiu testar, em cada período de agregação, a homogeneidade das estimativas das probabilidades de transição, ao longo dos anos de observação.

Escolhido o período de agregação, as estimativas das probabilidades de transição foram atribuídas às épocas correspondentes ao meio dos respectivos períodos. Embora esses períodos tenham sido escolhidos de tal forma que dentro deles as probabilidades de transição não apresentassem grandes discrepâncias, estabeleceu-se que as probabilidades diárias de transição deveriam

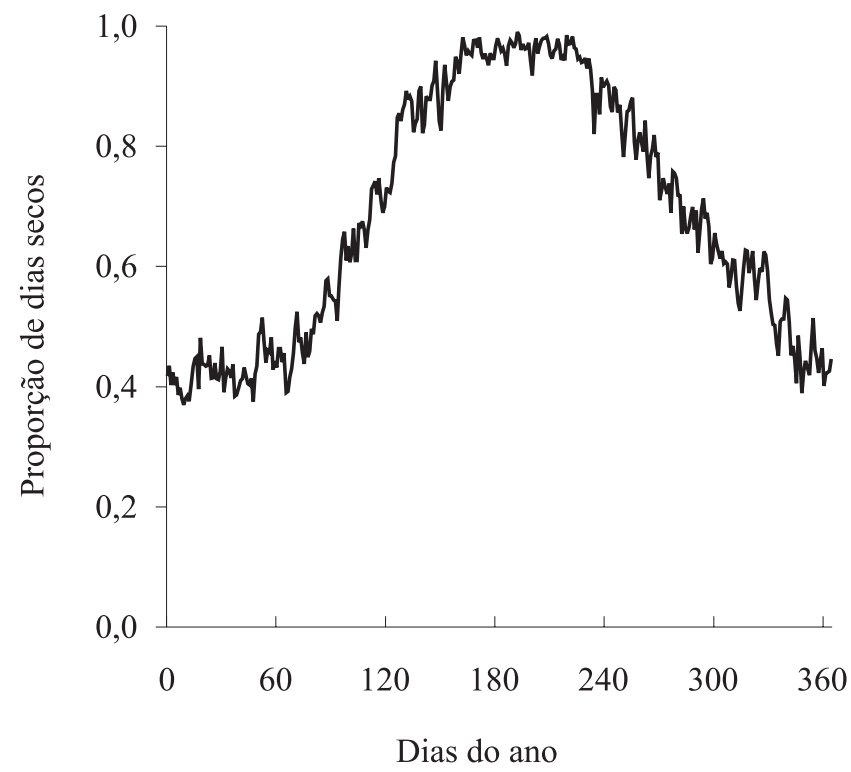

Figura 2. Evolução das proporções observadas de dias secos, em cada um dos 365 dias do ano, na Zona Homogênea A. apresentar pequenas diferenças ao longo de dias sucessivos. A acumulação inevitável dessas pequenas diferenças, em um período do tempo suficientemente longo, faz com que entre os diversos períodos de agregação as diferenças temporais entre as probabilidades de transição sejam consideráveis. Assim, as estimativas das probabilidades diárias de transição foram obtidas por interpolação de uma função, ajustada às estimativas das probabilidades de transição alocadas ao meio de cada período. Para isso, utilizou-se a função sinusoidal, definida pela equação (9). Como essa equação é uma função linear de seus parâmetros, utilizou-se o critério dos mínimos quadrados (Bloomfield, 1976) para estimar esses parâmetros.

A escassez de dados somente permite utilizar cadeias de Markov até a terceira ordem. A escolha da ordem baseou-se no teste das igualdades (5) e (6), com $r=0,1,2$ (Tabela 2). Estruturou-se esses testes de modo que permitissem verificar se as estimativas das probabilidades de transição são significativamente diferentes, entre os períodos anuais abrangidos pelos dados observados. Para efetuar esses testes, utilizou-se a análise de variância, por sua versatilidade na decomposição da variabilidade dos dados em mais de uma fonte de variação.

\section{Resultados e Discussão}

Os resultados iniciais do trabalho referem-se à escolha de um período adequado para a agregação dos dados. Mediante o uso dos estimadores (7) e (8), foram

Tabela 1. Grupos de meses, em cada período de agregação, definidos segundo a ordem do mês no período.

\begin{tabular}{|c|c|c|c|c|c|c|}
\hline Grupos de meses & & Períod & s de ag & gação & os dad & \\
\hline & & & Bir & stres & & \\
\hline $1^{\underline{0}}$ mês & Jan. & Mar. & Maio & Jul. & Set. & Nov. \\
\hline $2^{0}$ mês & Fev. & Abr. & Jun. & Ago. & Out. & Dez. \\
\hline & & & Tri & stres & & \\
\hline $1^{\underline{0}}$ mês & Jan. & Abr. & Jul. & Out. & & \\
\hline $2^{\underline{0}}$ mês & Fev. & Maio & Ago. & Nov. & & \\
\hline $3^{\underline{0}}$ mês & Mar. & Jun. & Set. & Dez. & & \\
\hline & & & Quad & nestre & & \\
\hline $1^{\underline{0}}$ mês & Jan. & Maio & Set. & & & \\
\hline $2^{\underline{0}}$ mês & Fev. & Jun. & Out. & & & \\
\hline $3^{0}$ mês & Mar. & Jul. & Nov. & & & \\
\hline $4^{\underline{0}}$ mês & Abr. & Ago. & Dez. & & & \\
\hline & & & Ser & stres & & \\
\hline $1^{\underline{0}}$ mês & Jan. & Jul. & & & & \\
\hline $2^{0}$ mês & Fev. & Ago. & & & & \\
\hline $3^{0}$ mês & Mar. & Set. & & & & \\
\hline $4^{\underline{0}}$ mês & Abr. & Out. & & & & \\
\hline $5^{\circ}$ mês & Maio & Nov. & & & & \\
\hline $6^{0}$ mês & Jun. & Dez. & & & & \\
\hline
\end{tabular}


estimadas todas as probabilidades de transição referentes a cadeias de Markov até a terceira ordem, correspondentes a agregações em períodos bimestrais, trimestrais, quadrimestrais, semestrais e anuais. Para essas estimativas, na Tabela 3 estão descritos os P-Valores referentes à aplicação da análise de variância, para testar a igualdade das probabilidades de transição dentro dos grupos de meses definidos na Tabela 1, o que corresponde a verificar se as probabilidades de transição não são muito discrepantes, dentro de cada período de agregação.

Examinando-se a Tabela 3, verifica-se que a hipótese de igualdade das probabilidades de transição, dentro de cada período considerado, somente pode ser sustentada, quando a agregação é feita em períodos de dois meses.

Tabela 2. Testes para a escolha da ordem da cadeia de Markov, até a terceira ordem.

\begin{tabular}{|c|c|c|}
\hline \multirow{2}{*}{$\begin{array}{l}\text { Testes de igualdade das } \\
\text { probabilidades de transição }\end{array}$} & \multicolumn{2}{|c|}{ Decisão quanto à hipótese de igualdade ${ }^{(1)}$} \\
\hline & Aceita-se $^{(2)}$ & Rejeita-se \\
\hline $\mathrm{P}(0 / 0)=\mathrm{P}(0 / 1)$ & $\mathrm{r}=0$ & $r \geq 1$ \\
\hline $\begin{array}{l}\mathrm{P}(0 / 00)=\mathrm{P}(0 / 10) \\
\mathrm{P}(0 / 01)=\mathrm{P}(0 / 11)\end{array}$ & $r=1$ & $r \geq 2$ \\
\hline $\begin{array}{l}\mathrm{P}(0 / 000)=\mathrm{P}(0 / 100) \\
\mathrm{P}(0 / 001)=\mathrm{P}(0 / 101) \\
\mathrm{P}(0 / 010)=\mathrm{P}(0 / 110) \\
\mathrm{P}(0 / 011)=\mathrm{P}(0 / 111)\end{array}$ & $\mathrm{r}=2$ & $\mathrm{r}=3$ \\
\hline
\end{tabular}

${ }^{(1)} \mathrm{r}$ : ordem da cadeia de Markov. ${ }^{(2)} \mathrm{r}=0$ : estados não correlacionados.

Tabela 3. P-Valores correspondentes aos testes $\mathrm{F}$ de igualdade das probabilidades de transição, dentro dos grupos de meses referidos na Tabela 1, segundo diversos períodos de agregação dos dados.

\begin{tabular}{|c|c|c|c|c|c|}
\hline \multirow{2}{*}{$\begin{array}{l}\text { Probabilidades } \\
\text { de transição }\end{array}$} & \multicolumn{5}{|c|}{ Períodos de agregação (meses) } \\
\hline & 12 & 6 & 4 & 3 & 2 \\
\hline \multirow[b]{2}{*}{$\mathrm{P}(0)$} & \multicolumn{5}{|c|}{ Cadeias de Markov de ordem zero } \\
\hline & $0,0000^{(1)}$ & $0,0000^{(1)}$ & 0,1164 & $0,0018^{(1)}$ & 0,6069 \\
\hline & \multicolumn{5}{|c|}{ Cadeias de Markov de primeira ordem } \\
\hline $\mathrm{P}(0 / 0)$ & $0,0000^{(1)}$ & $0,0002^{(1)}$ & 0,0536 & $0,0089^{(1)}$ & 0,6024 \\
\hline \multirow[t]{2}{*}{$\mathrm{P}(0 / 1)$} & $0,0000^{(1)}$ & $0,0247^{(1)}$ & $0,0449^{(1)}$ & 0,1545 & 0,6052 \\
\hline & \multicolumn{5}{|c|}{ Cadeias de Markov de segunda ordem } \\
\hline $\mathrm{P}(0 / 00)$ & $0,0000^{(1)}$ & $0,0027^{(1)}$ & 0,1419 & $0,0077^{(1)}$ & 0,9800 \\
\hline $\mathrm{P}(0 / 01)$ & $0,0000^{(1)}$ & 0,1318 & 0,1243 & 0,5651 & 0,4480 \\
\hline $\mathrm{P}(0 / 10)$ & $0,0000^{(1)}$ & $0,0003^{(1)}$ & $0,0186^{(1)}$ & 0,9919 & 0,4454 \\
\hline \multirow{2}{*}{$\mathrm{P}(0 / 11)$} & $0,0000^{(1)}$ & 0,7572 & 0,2787 & 0,4848 & 0,7466 \\
\hline & \multicolumn{5}{|c|}{ Cadeias de Markov de terceira ordem } \\
\hline $\mathrm{P}(0 / 000)$ & $0,0000^{(1)}$ & $0,0063^{(1)}$ & $0,0287^{(1)}$ & 0,0602 & 0,5248 \\
\hline $\mathrm{P}(0 / 001)$ & $0,0000^{(1)}$ & 0,1353 & 0,2092 & 0,9577 & 0,7292 \\
\hline $\mathrm{P}(0 / 010)$ & $0,0000^{(1)}$ & $0,0009^{(1)}$ & $0,0217^{(1)}$ & 0,5125 & 0,2993 \\
\hline $\mathrm{P}(0 / 011)$ & $0,0000^{(1)}$ & 0,1566 & $0,0272^{(1)}$ & 0,1738 & 0,8435 \\
\hline $\mathrm{P}(0 / 100)$ & $0,0000^{(1)}$ & $0,0256^{(1)}$ & 0,8138 & 0,1318 & 0,6312 \\
\hline $\mathrm{P}(0 / 101)$ & $0,0000^{(1)}$ & 0,1221 & 0,0844 & 0,6900 & 0,0643 \\
\hline $\mathrm{P}(0 / 110)$ & $0,0000^{(1)}$ & 0,0618 & 0,1034 & 0,5414 & 0,8051 \\
\hline $\mathrm{P}(0 / 111)$ & $0,0000^{(1)}$ & 0,9324 & 0,8403 & 0,7372 & 0,4080 \\
\hline
\end{tabular}

${ }^{(1)} \mathrm{P}$-Valores menores que $5 \%$.
A análise de variância permitiu, ainda, que fosse testada a homogeneidade das estimativas de cada probabilidade de transição, ao longo dos anos de observação. Os P-Valores desse teste, aplicado às estimativas obtidas nos diversos períodos de agregação, estão apresentados na Tabela 4. Verifica-se que as estimativas das diversas probabilidades de transição apresentam maior homogeneidade ao longo dos anos de observação, quando os dados são agregados em períodos bimestrais. Pode-se observar que nas agregações bimestrais todos os $\mathrm{P}$-Valores são superiores a $5 \%$, exceto o correspondente à probabilidade de transição de dia chuvoso para seco, que é igual a 4,51\%. Por essas razões, decidiu-se agregar os dados com base em períodos de dois meses.

Na Tabela 5, são descritas as estimativas das probabilidades de ocorrência de dia seco e as probabilidades de transição entre dias secos e chuvosos, para cadeias de Markov de ordens um, dois e três, obtidas com os estimadores (7) e (8), com base em dados agregados em períodos de dois meses.

Os valores dessas estimativas foram centrados em cada período bimestral e depois ajustados pela equação (9), utilizando-se o método dos mínimos quadrados. Na Tabela 6 apresentam-se, para cada probabilidade de transição, as estimativas dos parâmetros $\alpha, \beta$ e $\gamma$ da equação (9), bem como os valores dos respectivos coeficientes de determinação e erros quadráticos médios.

Tabela 4. P-Valores correspondentes à análise de variância para testar a igualdade das probabilidades de transição, entre os anos de observação, segundo os períodos de agregação dos dados.

\begin{tabular}{|c|c|c|c|c|c|}
\hline \multirow{2}{*}{$\begin{array}{l}\text { Probabilidades } \\
\text { de transição }\end{array}$} & \multicolumn{5}{|c|}{ Períodos de agregação (meses) } \\
\hline & 12 & 6 & 4 & 3 & 2 \\
\hline & \multicolumn{5}{|c|}{ Cadeias de Markov de ordem zero } \\
\hline $\mathrm{P}(0)$ & 0,1253 & 0,0804 & 0,1393 & $0,0407^{(1)}$ & 0,2828 \\
\hline & \multicolumn{5}{|c|}{ Cadeias de Markov de primeira ordem } \\
\hline $\mathrm{P}(0 / 0)$ & 0,3649 & 0,3408 & 0,3389 & 0,1272 & 0,4671 \\
\hline \multirow[t]{2}{*}{$\mathrm{P}(0 / 1)$} & $0,0001^{(1)}$ & $0,0003^{(1)}$ & $0,0035^{(1)}$ & $0,0078^{(1)}$ & $0,0451^{(1)}$ \\
\hline & \multicolumn{5}{|c|}{ Cadeias de Markov de segunda ordem } \\
\hline $\mathrm{P}(0 / 00)$ & 0,4742 & 0,5157 & 0,5337 & 0,2011 & 0,6207 \\
\hline $\mathrm{P}(0 / 01)$ & $0,0004^{(1)}$ & $0,0011^{(1)}$ & $0,0131^{(1)}$ & $0,0142^{(1)}$ & 0,1059 \\
\hline $\mathrm{P}(0 / 10)$ & 0,3403 & 0,3749 & 0,3645 & 0,2838 & 0,6841 \\
\hline $\mathrm{P}(0 / 11)$ & 0,1654 & 0,2216 & 0,2404 & 0,2483 & 0,4900 \\
\hline & \multicolumn{5}{|c|}{ Cadeias de Markov de terceira ordem } \\
\hline $\mathrm{P}(0 / 000)$ & 0,7729 & 0,8181 & 0,6424 & 0,7181 & 0,6977 \\
\hline $\mathrm{P}(0 / 001)$ & $0,0010^{(1)}$ & $0,0048^{(1)}$ & $0,0175^{(1)}$ & $0,0388^{(1)}$ & 0,1176 \\
\hline $\mathrm{P}(0 / 010)$ & 0,3197 & 0,3684 & 0,3061 & 0,2802 & 0,4568 \\
\hline $\mathrm{P}(0 / 011)$ & 0,1245 & 0,0803 & 0,1719 & 0,0501 & 0,4293 \\
\hline $\mathrm{P}(0 / 100)$ & 0,1486 & 0,2443 & 0,2647 & 0,0647 & 0,4964 \\
\hline $\mathrm{P}(0 / 101)$ & $0,0440^{(1)}$ & 0,0629 & 0,1429 & 0,1675 & 0,2153 \\
\hline $\mathrm{P}(0 / 110)$ & 0,5247 & 0,6533 & 0,5347 & 0,3633 & 0,7735 \\
\hline $\mathrm{P}(0 / 111)$ & 0,5115 & 0,5489 & 0,5053 & 0,6464 & 0,3837 \\
\hline
\end{tabular}

(1)P-Valores menores que 5\%. 
Tabela 5. Estimativas das probabilidades de transição para dia seco, na Zona Homogênea A, por ordem da cadeia de Markov, segundo períodos de dois meses.

\begin{tabular}{|c|c|c|c|c|c|c|c|}
\hline \multirow{2}{*}{$\begin{array}{l}\text { Ordem da cadeia } \\
\text { de } \text { Marcov }^{(1)}\end{array}$} & \multirow[t]{2}{*}{ Eventos $^{(2)}$} & \multicolumn{6}{|c|}{ Períodos } \\
\hline & & Jan./Fev. & Mar./Abr. & Maio/Jun. & Jul./Ago. & Set./Out. & Nov./Dez. \\
\hline Ordem 0 & $(0)$ & 0,4245 & 0,5714 & 0,9013 & 0,9471 & 0,7438 & 0,5176 \\
\hline \multirow[t]{2}{*}{ Ordem 1} & $(0 / 0)$ & 0,5471 & 0,6767 & 0,9220 & 0,9601 & 0,7870 & 0,6128 \\
\hline & $(0 / 1)$ & 0,3344 & 0,4198 & 0,6720 & 0,7453 & 0,6338 & 0,4216 \\
\hline \multirow[t]{4}{*}{ Ordem 2} & $(0 / 00)$ & 0,5949 & 0,7207 & 0,9274 & 0,9654 & 0,8075 & 0,6479 \\
\hline & $(0 / 01)$ & 0,4143 & 0,478 & 0,6930 & 0,7561 & 0,6519 & 0,4845 \\
\hline & $(0 / 10)$ & 0,4889 & 0,5673 & 0,8197 & 0,8584 & 0,7352 & 0,5633 \\
\hline & $(0 / 11)$ & 0,2938 & 0,3732 & 0,6028 & 0,7294 & 0,6087 & 0,3817 \\
\hline \multirow[t]{8}{*}{ Ordem 3} & $(0 / 000)$ & 0,6278 & 0,7444 & 0,9307 & 0,9684 & 0,8249 & 0,6681 \\
\hline & $(0 / 001)$ & 0,4379 & 0,4933 & 0,6974 & 0,7633 & 0,6633 & 0,5030 \\
\hline & $(0 / 010)$ & 0,5213 & 0,5999 & 0,8275 & 0,8750 & 0,7603 & 0,6031 \\
\hline & $(0 / 011)$ & 0,3554 & 0,4212 & 0,633 & 0,7286 & 0,6359 & 0,4429 \\
\hline & $(0 / 100)$ & 0,5452 & 0,6457 & 0,8412 & 0,9002 & 0,7587 & 0,6179 \\
\hline & $(0 / 101)$ & 0,3932 & 0,4520 & 0,6447 & 0,7177 & 0,6367 & 0,4630 \\
\hline & $(0 / 110)$ & 0,4671 & 0,5287 & 0,7740 & 0,8247 & 0,6979 & 0,5327 \\
\hline & $(0 / 111)$ & 0,2673 & 0,3394 & 0,5468 & 0,7143 & 0,5728 & 0,3512 \\
\hline
\end{tabular}

${ }^{(1)}$ Ordem 0: estados não correlacionados. ${ }^{(2)} 0=$ dia seco; 1 = dia chuvoso.

Tabela 6. Parâmetros das funções ajustantes das probabilidades de transição para dia seco.

\begin{tabular}{lcccccc}
\hline $\begin{array}{l}\text { Transições } \\
\text { de estado }\end{array}$ & \multicolumn{3}{c}{$\begin{array}{c}\text { Parâmetros da } \\
\text { curva ajustante }\end{array}$} & & \multicolumn{2}{c}{$\begin{array}{c}\text { Qualidade do } \\
\text { ajustamento }\end{array}$} \\
\cline { 2 - 3 } \cline { 6 - 7 } & $\alpha$ & $\beta$ & $\gamma$ & & $\mathrm{R}^{2(2)}$ & $\mathrm{EQM}^{(3)}$ \\
\hline$(0)$ & 0,68646 & $-0,25786$ & $-0,08279$ & & 0,9875 & 0,0031 \\
$(0 / 0)$ & 0,75266 & $-0,20552$ & $-0,05577$ & & 0,9908 & 0,0014 \\
$(0 / 1)$ & 0,53782 & $-0,19089$ & $-0,09809$ & & 0,9941 & 0,0009 \\
$(0 / 00)$ & 0,77853 & $-0,18551$ & $-0,04533$ & & 0,9948 & 0,0009 \\
$(0 / 01)$ & 0,57989 & $-0,15841$ & $-0,08044$ & & 0,9910 & 0,0011 \\
$(0 / 10)$ & 0,67327 & $-0,17871$ & $-0,07595$ & & 0,9827 & 0,0022 \\
$(0 / 11)$ & 0,49721 & $-0,19140$ & $-0,11319$ & & 0,9958 & 0,0007 \\
$(0 / 000)$ & 0,79460 & $-0,17318$ & $-0,04038$ & & 0,9974 & 0,0003 \\
$(0 / 001)$ & 0,59311 & $-0,14993$ & $-0,07857$ & & 0,9904 & 0,0009 \\
$(0 / 010)$ & 0,69921 & $-0,16452$ & $-0,07638$ & & 0,9863 & 0,0015 \\
$(0 / 011)$ & 0,53578 & $-0,16328$ & $-0,10170$ & & 0,9969 & 0,0004 \\
$(0 / 100)$ & 0,71976 & $-0,16415$ & $-0,06123$ & & 0,9903 & 0,0010 \\
$(0 / 101)$ & 0,55073 & $-0,14696$ & $-0,08488$ & & 0,9952 & 0,0004 \\
$(0 / 110)$ & 0,63830 & $-0,17154$ & $-0,07656$ & & 0,9805 & 0,0023 \\
$(0 / 111)$ & 0,46382 & $-0,18806$ & $-0,11822$ & & 0,9864 & 0,0022 \\
\hline
\end{tabular}

${ }^{(1)} 0$ : dia seco; 1: dia chuvoso. ${ }^{(2)}$ Coeficiente de determinação (proporção da variância explicada pela função ajustante). (3)Erro quadrático médio (média dos quadrados dos desvios entre os valores observados e os ajustados).

Estimados seus parâmetros, as funções ajustantes permitiram obter aproximações para todas as probabilidades diárias de transição, nas cadeias de Markov de ordem zero, um, dois e três, por meio de interpolação. A representação de probabilidades de transição por funções periódicas, definidas por séries de Fourier, é muito utilizada nos estudos climáticos (Stern \& Coe, 1984).

Na Figura 3, está descrita a evolução, ao longo de um período anual, das funções ajustantes das probabili- dades de transição para dia seco, nas cadeias de Markov de ordens zero e dois. Analogamente, a Figura 4 descreve a evolução dessas funções ajustantes, quando as cadeias de Markov são de ordem três. Nas Figuras 3 e 4 também estão destacadas as probabilidades de transição que foram atribuídas a cada período bimestral.

Conforme se pode observar nas Figuras 3 e 4, a evolução ao longo de um ano da função ajustante das probabilidades de ocorrência de dia seco e de transição para dia seco, nas cadeias de Markov até a terceira ordem, apresentam um mínimo no mês de janeiro e um máximo no mês de julho. Esse resultado é consistente com o fato de que, na região do Cerrado, existem duas estações bem distintas, em que o verão é essencialmente quente e chuvoso e o inverno é menos quente e seco (Vianello \& Alves, 1991). No Planalto Central brasileiro, o clima é caracterizado por uma época de seca de maio até setembro (Wolf, 1977).

A escolha da ordem da cadeia de Markov, mais adequada para a Zona A, foi efetuada utilizando-se a análise de variância, para testar a igualdade das diversas probabilidades de transição para dia seco, segundo os estados possíveis dos dias anteriores, de acordo com o roteiro descrito na Tabela 2 . Os resultados dessa análise, para a qual se adotou um nível de significância de 5\%, estão apresentados na Tabela 7. Verifica-se que uma cadeia de Markov de ordem zero não é adequada, uma vez que as probabilidades de transição para dia seco diferem significativamente nos casos em que o dia anterior é seco ou é chuvoso. 

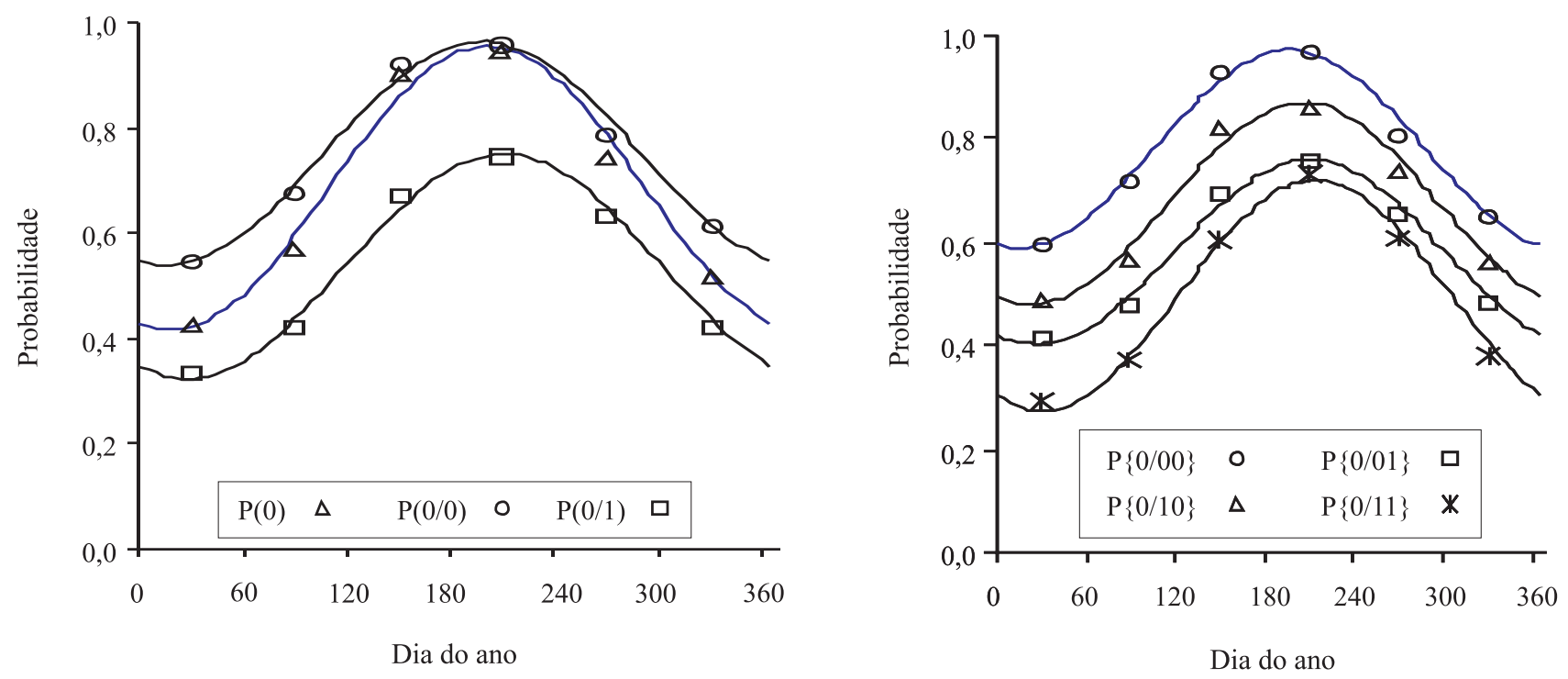

Figura 3. Ajustamento das probabilidades de transição para dia seco, nas cadeias de Markov: (A) ordens zero e um; (B) ordem dois.
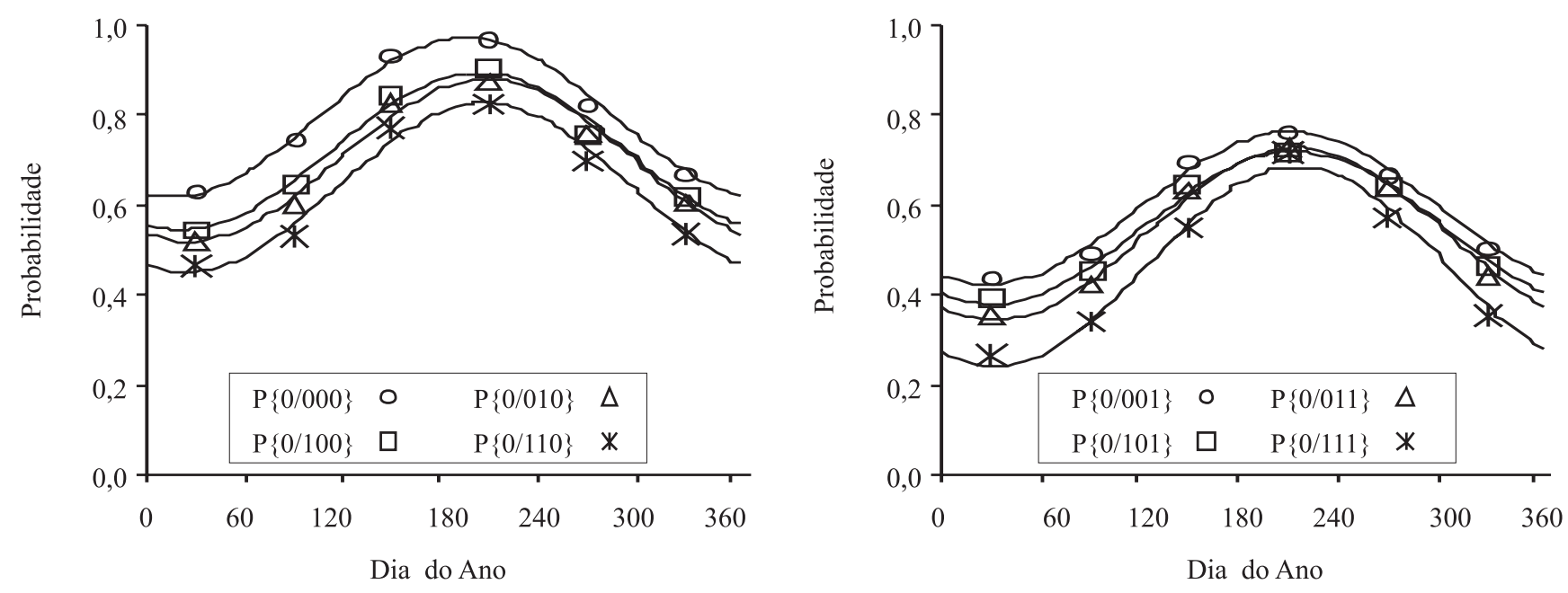

Figura 4. Ajustamento das probabilidades de transição para dia seco, nas cadeias de Markov: (A) ordem três, com dia anterior seco; (B) ordem três, com dia anterior chuvoso.

Verifica-se, ainda, que as cadeias de Markov de primeira ordem também não são adequadas, pois as probabilidades de transição para dia seco diferem significativamente quando se consideram os diversos estados possíveis nos dias anteriores.

A mesma conclusão se impõe para a cadeia de Markov de segunda ordem, tendo em vista que as probabilidades de transição para dia seco diferem significativamente quando se consideram todos os estados possíveis nos três dias anteriores.

A Tabela 7 permite constatar, também, que as estimativas de todas as probabilidades de transição diferem significativamente entre os seis períodos bimestrais definidos para a agregação dos dados.

Esses resultados evidenciam que na Zona A, do Planalto Central brasileiro, a ocorrência de um dia 
Tabela 7. Teste F da análise de variância para testar as igualdades entre várias probabilidades de transição para dia seco e entre os bimestres do ano, segundo a ordem da cadeia de Markov.

\begin{tabular}{|c|c|c|c|c|c|}
\hline \multirow{3}{*}{$\begin{array}{l}\text { Ordem da cadeia } \\
\text { de Markov }\end{array}$} & \multicolumn{5}{|c|}{ Testes de igualdade } \\
\hline & \multirow{2}{*}{$\begin{array}{l}\text { Probabilidades } \\
\text { de transição } \\
(1)\end{array}$} & \multicolumn{2}{|c|}{ Entre probabilidades ${ }^{(2)}$} & \multicolumn{2}{|c|}{ Entre bimestres ${ }^{(3)}$} \\
\hline & & $\mathrm{F}$ & P-Valor & $\mathrm{F}$ & P-Valor \\
\hline Ordem 1 & $\mathrm{P}(0 / 0)=\mathrm{P}(0 / 1)$ & 185,39 & 0,0000 & 75,06 & 0,0001 \\
\hline \multirow[t]{2}{*}{ Ordem 2} & $\mathrm{P}(0 / 00)=\mathrm{P}(0 / 10)$ & 86,40 & 0,0002 & 118,21 & 0,0000 \\
\hline & $\mathrm{P}(0 / 01)=\mathrm{P}(0 / 11)$ & 28,12 & 0,0032 & 67,95 & 0,0001 \\
\hline \multirow[t]{4}{*}{ Ordem 3} & $\mathrm{P}(0 / 000)=\mathrm{P}(0 / 100)$ & 110,53 & 0,0001 & 243,48 & 0,0000 \\
\hline & $\mathrm{P}(0 / 001)=\mathrm{P}(0 / 101)$ & 267,21 & 0,0000 & 1031,68 & 0,0000 \\
\hline & $\mathrm{P}(0 / 010)=\mathrm{P}(0 / 110)$ & 139,45 & 0,0001 & 920,34 & 0,0000 \\
\hline & $\mathrm{P}(0 / 011)=\mathrm{P}(0 / 111)$ & 34,67 & 0,0020 & 118,30 & 0,0000 \\
\hline
\end{tabular}

(1)0: dia seco; 1: dia chuvoso. (2)Valor crítico de F, a 5\% de significância: 6,61.(3)Valor crítico de F, a 5\% de significância: 5,05.

chuvoso é condicionalmente dependente da seqüência de dias secos e chuvosos nos três dias anteriores.

Portanto, as cadeias não-homogêneas de Markov de terceira ordem podem ser utilizadas, com vantagem, na análise probabilística da seqüência de dias secos e chuvosos, em regiões de clima assemelhado ao da Zona A do Planalto Central brasileiro.

Além de sua finalidade descritiva, as cadeias de Markov podem servir de base para a definição de um processo gerador, para simular seqüências de dias secos e chuvosos. O uso da simulação permite estimar distribuições de probabilidades de eventos, para os quais existem poucas observações (Koutsoyiannis \& Zarris, 1999). Como exemplo, a simulação de seqüências de dias secos e chuvosos, por meio de cadeias não-homogêneas de Markov, pode ser utilizada para a estimação e a análise das seguintes distribuições de probabilidades: número de estiagens de determinada duração, em certo período do tempo; número e duração de veranicos em determinada estação chuvosa; duração da maior estiagem em determinado período do tempo; duração de dias chuvosos consecutivos em determinada região; determinação do tempo de retorno de estiagens prolongadas.

\section{Conclusões}

1. Em uma grande zona pluviometricamente homogênea do Planalto Central brasileiro, a ocorrência de chuva, em cada dia, é condicionalmente dependente da seqüência de dias secos e chuvosos nos três dias anteriores.

2. A escassez de dados para se estimarem as probabilidades de transição, entre dias secos e chuvosos, pode ser contornada por meio da adequada agregação espacial e temporal dos dados.
3. As probabilidades diárias de transição entre dias secos e chuvosos podem ser obtidas por interpolação das probabilidades de transição, estimadas com dados agregados temporalmente, mediante o uso de uma função sinusoidal.

\section{Referências}

ANDRADE JÚNIOR, A.S.; FRIZZONE, J.A.; SENTELHAS, P.C. Simulação da precipitação diária para Parnaíba e Teresina, PI, em planilha eletrônica. Revista Brasileira de Engenharia Agrícola e Ambiental, v.5, p.271-278, 2001.

ASSAD, E.D.; SANO, E.E.J.; MASUTOMO, R.; CASTRO, L.H.; SILVA, F.A.M. Veranicos na região dos cerrados brasileiros: freqüências e probabilidades de ocorrência. Pesquisa Agropecuária Brasileira, v.28, p.993-1003, 1993.

ASSIS, F.N. Modelagem da ocorrência e da quantidade de chuva e de dias secos em Piracicaba-SP e Pelotas-RS. 1991. 134p. Tese (Doutorado) - Escola Superior de Agricultura Luiz de Queiroz, Piracicaba.

AYODE, J.O. Introdução à climatologia para os trópicos. Rio de Janeiro: Bertrand Brasil, 1983. 332p.

BACK, A.J. Aplicação de cadeias markovianas à modelagem da precipitação diária. Revista de Tecnologia e Ambiente, v.4, p.1728, 1997.

BLOOMFIELD, P. Fourier analysis of time series: an introduction. New York: Wiley, 1976. 258p.

DE GROEN, M.M.; SAVENIJE, H.H.G. Markov chains at daily timesteps, key to transpiration at monthly timesteps. In: WARFSA WATERNET SYMPOSIUM, 1., 2000, Maputo. Sustainable use of water resources: proceedings. Maputo: WARFSA, 2000. p.1-12.

GABRIEL, K.R.; NEUMANN, J. A Markov chain model for daily rainfall occurrence at Tel Aviv. Quarterly Journal of the Royal Meteorological Society, v.88, p.90-95, 1962.

GORDON, P. Théorie des chaînes de Markov finies et ses applications. Paris: Dunod, 1965. 130p. 
KELLER FILHO, T. Redução do risco climático na agricultura - uma abordagem probabilística. 1. Metodologia. In: SIMPÓSIO INTERNACIONAL DE SECURIDADE E ZONEAMENTO AGRÍCOLA DO MERCOSUL, 1., 1998, Brasília. Anais. Brasília: Ministério da Agricultura e Abastecimento, 1998. p.40-47.

KELLER FILHO, T.; ASSAD, E.D.; LIMA, P.R.S. de R. Regiões pluviometricamente homogêneas no Brasil. Pesquisa Agropecuária Brasileira, v.40, p.311-322, 2005.

KENDALL, M. Time series. $2^{\text {nd }}$ ed. London: Griffin, 1976. 197p. KOUTSOYIANNIS, D.; ZARRIS, D. Simulation of rainfall events for design purposes with inadequate data. In: GENERAL ASSEMBLY OF THE EUROPEAN GEOPHYSICAL SOCIETY, 24., 1999, The Hague. Proceedings. The Hague: European Geophysical Society, 1999. p.35-44.

LIMA, J.G.S. Gerenciamento de dados climatológicos heterogêneos em agricultura. 2003. 103p. Dissertação (Mestrado) - Universidade Estadual de Campinas, Campinas.

LIMA, P.R.S. de R. Redução do risco climático na agricultura uma abordagem probabilística - Resultados obtidos. In: SIMPÓSIO INTERNACIONAL DE SECURIDADE E ZONEAMENTO AGRÍCOLA DO MERCOSUL, 1., 1998, Brasília. Anais. Brasília: Ministério da Agricultura e Abastecimento, 1998. p.49-57.
MUHAMMAD, F.; NABI, G. Markov chain model for rainfall occurrence in Pakistan. Journal of Islamic Academy of Sciences, v.4, p.173-178, 1991.

PUNYAWARDENA, B.V.R.; KULASIRI, D. On development and comparative study of two Markov models of rainfall in the dry zone of Sri Lanka. In: JOINT INTERNATIONAL CONFERENCE ON AGRICULTURAL ENGINEERING AND TECHNOLOGY, 1997, Dhaka. Proceedings. Dhaka: ASAE, 1997. p.231-238.

SOUZA, S.A.V. de; PERES, F.C. Programa computacional para simulação da ocorrência de veranicos e queda de rendimento. Pesquisa Agropecuária Brasileira, v.33, p.1951-1956, 1998.

STERN, R.D.; COE, R. A model fitting analysis of daily rainfall data. Journal of the Royal Statistical Society, v.147, p.1-34, 1984. STERN, R.D.; COE, R. The use of rainfall models in agricultural planning. Agricultural Meteorology, v.26, p.35-50, 1982.

TUCCI, C.E.M. Hidrologia: ciência e aplicação. Porto Alegre: Ed. da UFRGS, 2004.943p.

VIANELLO, R.L.; ALVES, A.R. Meteorologia básica e aplicações. Viçosa: Imprensa Universitária, Universidade Federal de Viçosa, 1991.449p.

WOLF, J.M. Probabilidade de ocorrência de períodos secos na estação chuvosa de Brasília. Pesquisa Agropecuária Brasileira, v.12, p.141-150, 1977.

Recebido em 18 de maio de 2005 e aprovado em 9 de junho de 2006 